\title{
Stabilizing Minimum Error Rate Training
}

\author{
George Foster and Roland Kuhn \\ National Research Council Canada \\ first.last@nrc.gc.ca
}

\begin{abstract}
The most commonly used method for training feature weights in statistical machine translation (SMT) systems is Och's minimum error rate training (MERT) procedure. A well-known problem with Och's procedure is that it tends to be sensitive to small changes in the system, particularly when the number of features is large. In this paper, we quantify the stability of Och's procedure by supplying different random seeds to a core component of the procedure (Powell's algorithm). We show that for systems with many features, there is extensive variation in outcomes, both on the development data and on the test data. We analyze the causes of this variation and propose modifications to the MERT procedure that improve stability while helping performance on test data.
\end{abstract}

\section{Introduction}

Most recent approaches in SMT, eg (Koehn et al., 2003; Chiang, 2005), use a log-linear model to combine probabilistic features. Minimum ErrorRate Training (MERT) aims to find the set of loglinear weights that yields the best translation performance on a development corpus according to some metric such as BLEU. This is an essential step in SMT training that can significantly improve performance on a test corpus compared to setting weights by hand. MERT is a difficult problem, however, because calculating BLEU as a function of log-linear weights requires decoding, which is an expensive operation. Moreover, because this function is not differentiable, efficient gradient-based optimization algorithms cannot be used.

Och's procedure is the most widely-used version of MERT for SMT (Och, 2003). To reduce computational cost, it relies on the key technique of optimizing weights over n-best lists of translation hypotheses rather than over all possible hypotheses. This allows the most probable hypothesis under a given set of weights - and the corresponding BLEU score- to be found by enumerating n-best entries rather than decoding. Some variant on Powell's algorithm (Press et al., 2002) is typically used to maximize BLEU in this setting. The n-best lists are constructed by alternating decoding and BLEU maximization operations: decoding adds new hypotheses to the current lists, then BLEU is maximized over the lists to find new best weights for the subsequent decoding step, etc. This process continues until no new hypotheses are found.

Och's procedure works well in practice, usually converging after 10-20 calls to the decoder, far fewer than would be required to maximize BLEU directly with a general-purpose optimization algorithm. However, it tends to be sensitive to small changes in the system, particularly for large feature sets. This is a well-known problem with Och's procedure (Och et al., 2004). It makes it difficult to assess the contribution of features, because the measured gain in performance due to a new feature can depend heavily on the setting of some apparently unrelated parameter such as the size of n-best list used. Features with the potential for statistically significant gains may be rejected because Och's procedure failed to find good weights for them.

In this paper we attempt to quantify the stability of Och's procedure under different conditions by measuring the variation in test-set scores across different random seeds used with Powell's algorithm. We show that there is extensive variation for large feature sets, and that it is due to two main factors: the occasional failure of Och's procedure to find a good maximum on the development set, and the failure of some maxima to generalize to 
the test set. We analyze the causes of each of these problems, and propose solutions for improving the stability of the overall procedure.

\section{Previous Work}

One possible approach to estimating log-linear weights on features is to dispense with the n-best lists employed by Och's procedure and, instead, to optimize weights by directly accessing the decoder. The disadvantage of this approach is that far more iterations of decoding of the full development set are required. In (Zens and Ney, 2004) the downhill simplex method is used to estimate the weights; around 200 iterations are required for convergence to occur. However, each iteration is unusually fast, because only monotone decoding is permitted (i.e., the order of phrases in the target language mirrors that in the source language). Similarly, Cettolo and Federico (2004) apply the simplex method to optimize weights directly using the decoder. In their experiments on NIST 2003 Chinese-English data, they found about 100 iterations of decoding were required. Although they obtained consistent and stable performance gains for MT, these were inferior to the gains yielded by Och's procedure in (Och, 2003). Taking Och's MERT procedure as a baseline, (Zens et al., 2007) experiment with different training criteria for SMT and obtain the best results for a criterion they call "expected BLEU score".

Moore and Quirk (2008) share the goal underlying our own research: improving, rather than replacing, Och's MERT procedure. They focus on the step in the procedure where the set of feature weights optimizing BLEU (or some other MT metric) for an n-best list is estimated. Typically, several different starting points are tried for this set of weights; often, one of the starting points is the best set of weights found for the previous set of n-best hypotheses. The other starting points are often chosen randomly. In this paper, Moore and Quirk look at the best way of generating the random starting points; they find that starting points generated by a random walk from previous maxima are superior to those generated from a uniform distribution. The criterion used throughout the paper to judge the performance of MERT is the BLEU score on the development test set (rather than, for instance, the variance of that score, or the BLEU score on held-out test data). Another contribution of the paper is ingenious methods for pruning the set of n-best hypotheses at each iteration.

Cer et al (2008) also aim at improving Och's MERT. They focus on the search for the best set of weights for an n-best list that follows choice of a starting point. They propose a modified version of Powell's in which "diagonal" directions are chosen at random. They also modify the objective function used by Powell's to reflect the width of the optima found. They are able to show that their modified version of MERT outperforms both a version using Powell's, and a more heuristic search algorithm devised by Philipp Koehn that they call Koehn Coordinate Descent, as measured on the development set and two test data sets. (Duh and Kirchhoff, 2008) ingeniously uses MERT as a weak learner in a boosting algorithm that is applied to the n-best reranking task, with good results (a gain of about $0.8 \mathrm{BLEU}$ on the test set).

Recently, some interesting work has been done on what might be considered a generalization of Och's procedure (Macherey et al., 2008). In this generalization, candidate hypotheses in each iteration of the procedure are represented as lattices, rather than as n-best lists. This makes it possible for a far greater proportion of the search space to be represented: a graph density of 40 arcs per phrase was used, which corresponds to an n-best size of more than two octillion $\left(2 * 10^{27}\right)$ entries. Experimental results for three NIST 2008 tasks were very encouraging: though BLEU scores for the lattice variant of Och's procedure did not typically exceed those for the n-best variant on development data, on test data the lattice variant outperformed the n-best approach by between 0.6 and 2.5 BLEU points. The convergence behaviour of the lattice variant was also much smoother than that of the n-best variant. It would be interesting to apply some of the insights of the current paper to the lattice variant of Och's procedure.

\section{Och's MERT Procedure}

Och's procedure works as follows. First the decoder is run using an initial set of weights to generate $n$ best translations (usually around 100) for each source sentence. These are added to existing n-best lists (initially empty). Next, Powell's algorithm is used to find the weights that maximize BLEU score when used to choose the best hypotheses from the n-best lists. These weights 
are plugged back into the decoder, and the process repeats, nominally until the n-best lists stop growing, but often in practice until some criterion of convergence such as minimum weight change is attained. The weights that give the best BLEU score when used with the decoder are output.

The point of this procedure is to bypass direct search for the weights that result in maximum BLEU score, which would involve decoding using many different sets of weights in order to find which ones gave the best translations. Och's procedure typically runs the decoder only 10-20 times, which is probably at least one order of magnitude fewer than a direct approach. The main trick is to build up n-best lists that are representative of the search space, in the sense that a given set of weights will give approximately the same BLEU score when used to choose the best hypotheses from the n-best lists as it would when decoding. By iterating, the algorithm avoids weights that give good scores on the n-best lists but bad ones with the decoder, since the bad hypotheses that are scored highly by such weights will get added to the n-best lists, thereby preventing the choice of these weights in future iterations. Unfortunately, there is no corresponding guarantee that weights which give good scores with the decoder but bad ones on the nbest lists will get chosen.

Finding the set of weights that maximizes BLEU score over n-best lists is a relatively easy problem because candidate weight sets can be evaluated in time proportional to $n$ (simply calculate the score of each hypothesis according to the current weight set, then measure BLEU on the highest scoring hypothesis for each source sentence). Powell's algorithm basically loops over each feature in turn, setting its weight to an optimum value before moving on. ${ }^{1}$ Och's linemax algorithm is used to perform this optimization efficiently and exactly. However this does not guarantee that Powell's algorithm will find a global maximum, and so Powell's is typically run with many different randomly-chosen initial weights in order to try to find a good maximum.

\section{Experimental Setup}

The experiments described here were carried out with a standard phrase-based SMT system (Koehn

\footnotetext{
${ }^{1}$ It can also choose to optimize linear combinations of weights in order to avoid ridges that are not aligned with the original coordinates, which can be done just as easily.
}

\begin{tabular}{|l|r|r|}
\hline corpus & num sents & num Chinese toks \\
\hline dev1 & 1506 & 38,312 \\
dev2 & 2080 & 55,159 \\
nist04 & 1788 & 53,446 \\
nist06 & 1664 & 41,798 \\
\hline
\end{tabular}

Table 1: Development and test corpora.

et al., 2003) employing a log-linear combination of feature functions. HMM and IBM2 models were used to perform separate word alignments, which were symmetrized by the usual "diag-and" algorithm prior to phrase extraction. Decoding used beam search with the cube pruning algorithm (Huang and Chiang, 2007).

We used two separate log-linear models for MERT:

- large: 16 phrase-table features, 24 -gram language model features, 1 distortion feature, and 1 word-count feature (20 features in total).

- small: 2 phrase-table features, 14 -gram language model feature, 1 distortion feature, and 1 word-count feature (5 features in total).

The phrase-table features for the large model were derived as follows. Globally-trained HMM and IBM2 models were each used to extract phrases from UN and non-UN portions of the training corpora (see below). This produced four separate phrase tables, each of which was used to generate both relative-frequency and "lexical" conditional phrase-pair probabilities in both directions (target given source and vice versa). The two language model features in the large log-linear model were trained on the UN and non-UN corpora. Phrasetable features for the small model were derived by taking the union of the four individual tables, summing joint counts, then calculating relative frequencies.

All experiments were run using the Chinese/English data made available for NIST's 2008 MT evaluation. This included approximately $5 \mathrm{M}$ sentence pairs of data from the UN corpus, and approximatel 4M sentence pairs of other material. The English Gigaword corpus was not used for language model training. Two separate development corpora were derived from a mix of the NIST 2005 evaluation set and some webtext drawn from the training material (disjoint from the training set used). The evaluation sets for NIST 2004 


\begin{tabular}{|l|rrr|rrr|}
\hline cfg & \multicolumn{3}{|c|}{ nist04 } & \multicolumn{3}{c|}{ nist06 } \\
& avg & $\Delta$ & $S$ & avg & $\Delta$ & $S$ \\
\hline S1 & 31.17 & 1.09 & 0.28 & 26.95 & 0.90 & 0.27 \\
S2 & 31.44 & 0.22 & 0.07 & 27.38 & 0.71 & 0.19 \\
L1 & 33.03 & 1.09 & 0.37 & 29.22 & 0.97 & 0.34 \\
L2 & 33.37 & 1.49 & 0.49 & 29.61 & 2.14 & 0.66 \\
\hline
\end{tabular}

Table 2: Test-set BLEU score variation with 10 different random seeds, for small (S) and large (L) models on dev sets 1 and 2. The avg column gives the average BLEU score over the 10 runs; $\Delta$ gives the difference between the maximum and minimum scores, and $S$ is the standard deviation.

and NIST 2005 corpora were used for testing. Table 1 summarizes the sizes of the devtest corpora, all of which have four reference translations.

\section{Measuring the Stability of Och's Algorithm}

To gauge the response of Och's algorithm to small changes in system configuration, we varied the seed value for initializing the random number generator used to produce random starting points for Powell's algorithm. For each of 10 different seed values, Och's algorithm was run for a maximum of 30 iterations $^{2}$ using 100 -best lists. Table 2 shows the results for the two different log-linear models described in the previous section.

The two development sets exhibit a similar pattern: the small models appear to be somewhat more stable, but all models show considerable variation in test-set BLEU scores. For the large models, the average difference between best and worst BLEU scores is almost $1.5 \%$ absolute, with an average standard deviation of almost $0.5 \%$. Differences of as little as $0.35 \%$ are significant at a $95 \%$ confidence level according to paired bootstrap resampling tests on this data, so these variations are much too large to be ignored.

The variation in table 2 might result from Och's algorithm failing to maximize development-set BLEU properly on certain runs. Alternatively, it could be finding different maxima that vary in the extent to which they generalize to the test sets. Both of these factors appear to play a role. The ranges of BLEU scores on the two development corpora with the large models are 0.86 and 1.3 respectively; the corresponding standard deviations

\footnotetext{
${ }^{2}$ Sufficient for effective convergence in all cases we ${ }^{2} \mathrm{Su}$
tested.
}

\begin{tabular}{|l|rr|rr|r|}
\hline dev & \multicolumn{2}{|c|}{ nist04 } & \multicolumn{2}{|c|}{ nist06 } & inter \\
& $\rho$ & $r$ & $\rho$ & $r$ & $\rho$ \\
\hline dev1 & 0.18 & 0.42 & -0.27 & 0.07 & 0.73 \\
dev2 & 0.55 & 0.60 & 0.73 & 0.85 & 0.94 \\
\hline
\end{tabular}

Table 3: Pearson $(\rho)$ and Spearman rank $(r)$ correlation between dev-set and test-set BLEU scores for the large log-linear model. The final column shows nist04/nist06 correlation.

are 0.27 and 0.38 . Different runs clearly have significantly different degrees of success in maximizing BLEU.

To test whether the variation in developmentset BLEU scores accounts completely for the variation in test-set scores, we measured the correlation between them. The results in table 3 show that this varies considerably across the two development and test corpora. Although the rank correlation is always positive and is in some cases quite high, there are many examples where higher development-set scores lead to lower testset scores. Interestingly, the correlation between the two test-set scores (shown in the last column of the table) is much higher than that between the development and test sets. Since the test sets are not particularly similar to each other, this suggests that some sets of log-linear weights are in fact overfitting the development corpus.

\subsection{Bootstrapping with Random Seeds}

The results above indicate that the stability problems with Och's MERT can be quite severe, especially when tuning weights for a fairly large number of features. However, they also constitute a baseline solution to these problems: run MERT some number of times with different random seeds, then choose the run that achieves the highest BLEU score on a test set. Since testset scores are highly correlated, these weights are likely to generalize well to new data. Applying this procedure using the nist04 corpus to choose weights yields a BLEU increase of 0.69 on nist06 compared to the average value over the 10 runs in table 2; operating in the reverse direction gives an increase of 0.37 on nist $04 .{ }^{3}$

\footnotetext{
${ }^{3}$ These increases are averages over the increases on each development set. This comparison is not strictly fair to the baseline single-MERT procedure, since it relies on a test set for model selection (using the development set would have yielded gains of 0.25 for nist06 and 0.27 for nist04). However, it is fairly typical to select models (involving different feature sets, etc) using a test set, for later evaluation on a
} 


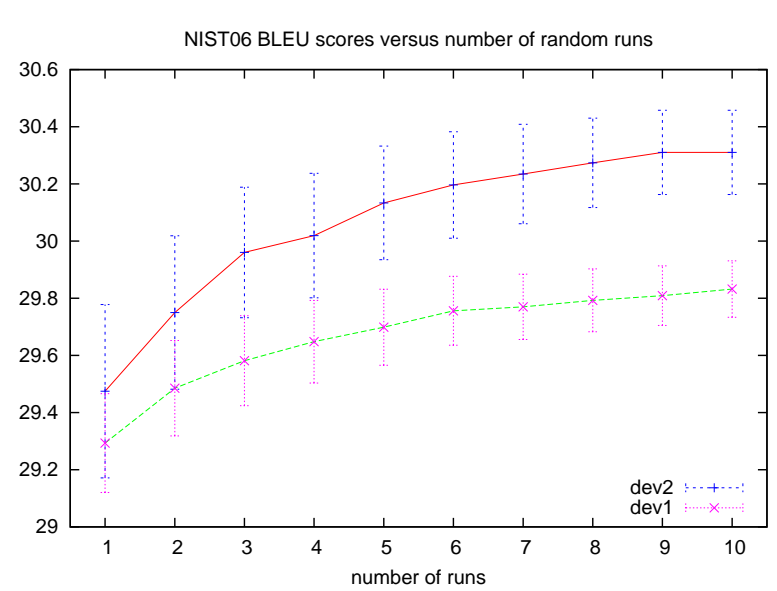

Figure 1: Results on the nist06 test corpus, using nist04 to choose best weights from varying numbers of MERT runs, averaged over 1000 random draws. The error bars indicate the magnitude of the standard deviation.

An obvious drawback to this technique is that it requires the expensive MERT procedure to be run many times. To measure the potential gain from using fewer runs, and to estimate the stability of the procedure, we used a bootstrap simulation. For each development set and each $n$ from 1 to 10 , we randomly drew 1000 sets of $n$ runs from the data used for table 2, then recorded the behaviour of the nist06 scores that corresponded to the best nist04 score. The results are plotted in figure 1 . There is no obvious optimal point on the curves, although 7 runs would be required to reduce the standard deviation on dev2 (the set with the higher variance) below 0.35 . In the following sections we evaluate some alternatives that are less computationally expensive. The large model setting is assumed throughout.

\section{Improving Maximization}

In this section we address the problem of improving the maximization procedure over the development corpus. In general, we expect that being able to consistently find higher maxima will lead to lower variance in test-set scores. Previous work, eg (Moore and Quirk, 2008; Cer et al., 2008), has focused on improving the performance of Powell's algorithm. The degree to which this is effective depends on how good an approximation the current n-best lists are to the true search space. As illus-

second, blind, test set. A multi-MERT strategy could be naturally incorporated into such a regime, and seems unlikely to give rise to substantial bias.

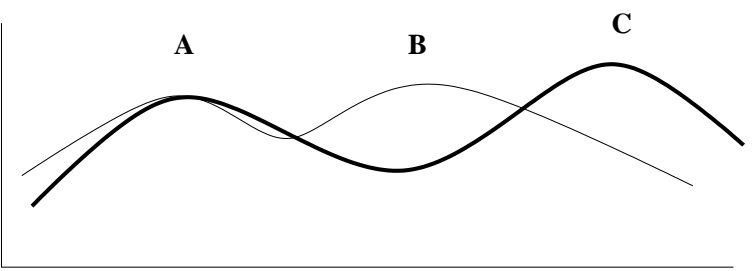

Figure 2: True objective function (bold curve) compared to n-best approximation (light curve). Och's algorithm can correct for false maxima like $\mathrm{B}$ by adding hypotheses to n-best lists, but may not find the true global maximum (C), converging to local peaks like A instead.

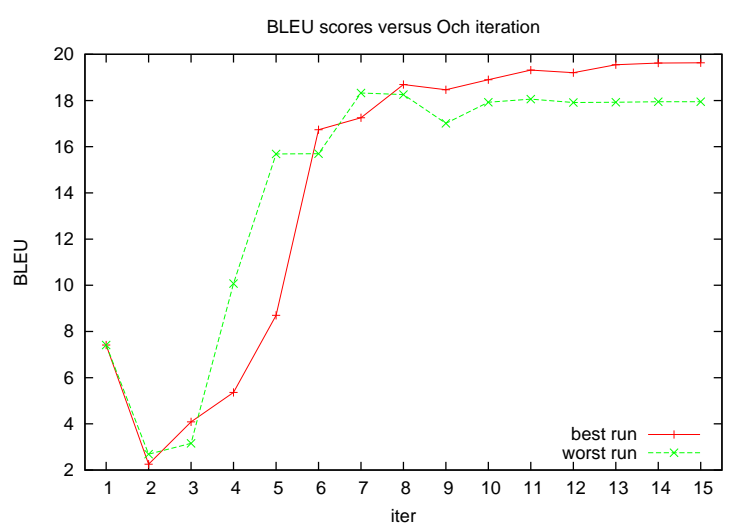

Figure 3: Development-set BLEU scores after each Och iteration for two different training runs on the dev 2 corpus.

trated in figure 2, it is possible for the true space to contain maxima that are absent from the approximate (n-best) space. Figure 3 gives some evidence that this happens in practice. It shows the evolution of decoder BLEU scores with iteration for the best and worst runs for dev2. Although the worst run explores a somewhat promising area at iteration 7 , it converges soon afterwards in a region that gives lower true BLEU scores. This is not due to a failure of Powell's algorithm, since the scores on the n-best lists rise monotonically in this range.

We explored various simple strategies for avoiding the kind of local-maximum behaviour exhibited in figure 3 . These are orthogonal to improvements to Powell's algorithm, which was used in its standard form. Our baseline implementation of Och's algorithm calls Powell's three times starting with each of the three best weight sets from the previous iteration, then a certain number of times with randomly-generated weights. The total number of Powell's calls is determined by an algorithm that tries to minimize the probability of 
a new starting point producing a better maximum. ${ }^{4}$

The first strategy was simply to re-seed the random number generator (based on a given global seed value) for each iteration of Och's algorithm. Our implementation had previously re-used the same "random" starting points for Powell's across different Och iterations. This is arguably justifiable on the grounds that the function to be optimized is different each time.

The second strategy was motivated by the observation that after the first several iterations of Och's algorithm, the starting point that leads to the best Powell's result is nearly always one of the three previous best weight sets rather than a randomly-generated set. To encourage the algorithm to consider other alternatives, we used the three best results from all previous Och's iterations. That is, on iteration $n$, Powell's is started with the three best results from iteration $n-1$, then the three best from $n-2$, and so forth. If more than $3(n-1)$ points are required by the stopping algorithm described above, then they are generated randomly.

The final strategy is more explicitly aimed at forcing the algorithm to cover a broader portion of the search space. Rather than choosing the maximum-BLEU results from Powell's algorithm for the subsequent decoding step, we choose weight vectors that yield high BLEU scores and are dissimilar from previous decoding weights. Formally:

$$
\hat{\alpha}=\underset{\alpha \in \mathcal{P}}{\operatorname{argmax}} w \operatorname{rbleu}(\alpha)+(1-w) \operatorname{rdist}(\alpha),
$$

where $\mathcal{P}$ is the set of all weight vectors returned by Powell's on the current iteration, $\operatorname{rbleu}(\alpha)$ is $\alpha$ 's BLEU score divided by the highest score for any vector in $\mathcal{P}$, and $\operatorname{rdist}(\alpha)$ is $\alpha$ 's distance to previous weights divided by the largest distance for any vector in $\mathcal{P}$. Distance to previous weights is measured by taking the minimum L2 distance from $\alpha$ to any of the decoding weight vectors used during the previous $m$ Och iterations.

Intuitively, the weight $w$ that controls the importance of BLEU score relative to novelty should increase gradually as Och's algorithm progresses in order to focus the search on the best maxi-

\footnotetext{
${ }^{4}$ Whenever a new maximum is encountered, at least the current number of new starting points must be tried before stopping, with a minimum of 10 points in total. Experiments where the total number of starts was fixed at 30 did not produce significantly different results.
}

mum found (roughly similar to simulated annealing search). To accomplish this, $w$ is defined as:

$$
w=1-a /(\text { iter }+b)
$$

where $b \geq 0$ and $a \leq b+1$ are parameters that control $w$ 's decay, and iter is the current Och iteration.

Each of the three strategies outlined above was run using 10 random seeds with both development corpora. The weight selection strategy was run with two different sets of values for the $a$ and $b$ parameters: $a=1, b=1$ and $a=5, b=9$. Each assigns equal weight to BLEU score and novelty on the first iteration, but under the first parameterization the weight on novelty decays more swiftly, to 0.03 by the final iteration compared to to 0.13 .

The results are shown in table 4 . The best strategy overall appears to be a combination of all three techniques outlined above. Under the $a=5$, $b=9, m=3$ parametrization for the final (weight selection) strategy, this improves the development set scores by an average of approximately $0.4 \%$ BLEU compared to the baseline, while significantly reducing the variation across different runs. Performance of weight selection appears to be quite insensitive to its parameters: there is no significant difference between the $a=1, b=1$ and $a=5, b=9$ settings. It is possible that further tuning of these parameters would yield better results, but this is an expensive procedure; we were also wary of overfitting. A good fallback is the first two strategies, which together achieve results that are almost equivalent to the final gains due to weight selection.

\section{Generalization}

As demonstrated in section 5, better performance on the development set does not necessarily lead to better performance on the test set: two weight vectors that give approximately the same dev-set BLEU score can give very different test-set scores. We investigated several vectors with this characteristic from the experiments described above, but were unable to find any intrinsic property that was a good predictor of test-set performance, perhaps due to the fact that the weights are scale invariant. We also tried averaging BLEU over bootstrapped samples of the development corpora, but this was also not convincingly correlated with testset BLEU. 


\begin{tabular}{|ll|rrr|}
\hline strategy & dev & avg & $\Delta$ & $S$ \\
\hline baseline & 1 & 22.64 & 0.87 & 0.27 \\
& 2 & 19.11 & 1.31 & 0.38 \\
re-seed & 1 & 22.87 & 0.65 & 0.21 \\
& 2 & 19.37 & 0.60 & 0.17 \\
+history & 1 & 22.99 & 0.43 & 0.15 \\
& 2 & 19.44 & 0.35 & 0.11 \\
+ sel 1,1,3 & 1 & 23.12 & 0.59 & 0.19 \\
& 2 & 19.53 & 0.38 & 0.13 \\
+sel 5,9,3 & 1 & 23.11 & 0.42 & 0.13 \\
& 2 & 19.46 & 0.44 & 0.14 \\
\hline
\end{tabular}

Table 4: Performance of various strategies for improving maximization on the dev corpora: baseline is the baseline used in section 5; re-seed is random generator re-seeding; history is accumulation of previous best weights as starting point; and sel $a, b, m$ is the final, weight selection, strategy described in section 6 , parameterized by $a, b$, and $m$. Strategies are applied cumulatively, as indicated by the + signs.

An alternate approach was inspired by the regularization method described in (Cer et al., 2008). In essence, this uses the average BLEU score from the points close to a given maximum as a surrogate for the BLEU at the maximum, in order to penalize maxima that are "narrow" and therefore more likely to be spurious. While Cer et al use this technique while maximizing along a single dimension within Powell's algorithm, we apply it over all dimensions with the vectors output from Powell's. Each individual weight is perturbed according to a normal distribution (with variance 1e-03), then the resulting vector is used to calculate BLEU over the n-best lists. The average score over 10 such perturbed vectors is used to calculate rbleu in the weight-selection method from the previous section.

The results from regularized weight selection are compared to standard weight selection and to the baseline MERT algorithm in table 5. Regularization appears to have very little effect on the weight selection approach. This does not necessarily contradict the results of Cer et al, since it is applied in a very different setting. The standard weight selection technique (in combination with the re-seeding and history accumulation strategies) gives a systematic improvement in average test-set BLEU score over the baseline, although it does not substantially reduce variance.

\begin{tabular}{|lll|rrr|}
\hline strategy & dev & test & avg & $\Delta$ & $S$ \\
\hline baseline & 1 & 04 & 33.03 & 1.09 & 0.37 \\
& & 06 & 29.22 & 0.97 & 0.34 \\
& 2 & 04 & 33.37 & 1.49 & 0.49 \\
& & 06 & 29.61 & 2.14 & 0.66 \\
\hline (+) sel 5,9,3 & 1 & 04 & 33.43 & 1.23 & 0.41 \\
& & 06 & 29.62 & 0.98 & 0.31 \\
& 2 & 04 & 33.95 & 1.03 & 0.37 \\
& & 06 & 30.32 & 0.88 & 0.30 \\
\hline + reg 10 & 1 & 04 & 33.36 & 1.45 & 0.49 \\
& & 06 & 29.56 & 1.25 & 0.39 \\
& 2 & 04 & 33.81 & 0.94 & 0.28 \\
& & 06 & 30.17 & 1.21 & 0.35 \\
\hline
\end{tabular}

Table 5: Performance of various MERT techniques on the test corpora. (+) sel 5,9,3 is the same configuration as + sel 5,9,3 in table $4 ;+$ reg 10 uses regularized BLEU within this procedure.

\section{Conclusion}

In this paper, we have investigated the stability of Och's MERT algorithm using different random seeds within Powell's algorithm to simulate the effect of small changes to a system. We found that test-set BLEU scores can vary by 1 percent or more across 10 runs of Och's algorithm with different random seeds. Using a bootstrap analysis, we demonstrate that an effective, though expensive, way to stabilize MERT would be to run it many times (at least 7), then choose the weights that give best results on a held-out corpus. We propose less expensive simple strategies for avoiding local maxima that systematically improve testset BLEU scores averaged over 10 MERT runs, as well as reducing their variance in some cases. An attempt to improve on these strategies by regularizing BLEU was not effective.

In future work, we plan to integrate improved variants on Powell's algorithm, which are orthogonal to the investigations reported here.

\section{Acknowlegement}

This material is partly based upon work supported by the Defense Advanced Research Projects Agency (DARPA) under Contract No. HR001106-C-0023. Any opinions, findings and conclusions or recommendations expressed in this material are those of the authors and do not necessarily reflect the views of the Defense Advanced Research Projects Agency (DARPA). 


\section{References}

Daniel Cer, Daniel Jurafsky, and Christopher D. Manning. 2008. Regularization and search for minimum error rate training. In Proceedings of the ACL Workshop on Statistical Machine Translation, Columbus, June. WMT.

Mauro Cettolo and Marcello Federico. 2004. Minimum error training of log-linear translation models. In International Workshop on Spoken Language Translation, Kyoto, September.

David Chiang. 2005. A hierarchical phrase-based model for statistical machine translation. In Proceedings of the 43th Annual Meeting of the Association for Computational Linguistics (ACL), Ann Arbor, Michigan, July.

Kevin Duh and Katrin Kirchhoff. 2008. Beyond loglinear models: Boosted minimum error rate training for n-best re-ranking. In Proceedings of the 46th Annual Meeting of the Association for Computational Linguistics (ACL), Columbus, Ohio, June.

Liang Huang and David Chiang. 2007. Forest rescoring: Faster decoding with integrated language models. In Proceedings of the 45th Annual Meeting of the Association for Computational Linguistics $(A C L)$, Prague, Czech Republic, June.

Philipp Koehn, Franz Josef Och, and Daniel Marcu. 2003. Statistical phrase-based translation. In Eduard Hovy, editor, Proceedings of the Human Language Technology Conference of the North American Chapter of the Association for Computational Linguistics, pages 127-133, Edmonton, Alberta, Canada, May. NAACL.

Wolfgang Macherey, Franz Josef Och, Ignacio Thayer, and Jakob Uszkoreit. 2008. Lattice-based minimum error rate training for statistical machine translation. In Proceedings of the 2008 Conference on Empirical Methods in Natural Language Processing (EMNLP), Honolulu.

Robert C. Moore and Chris Quirk. 2008. Random restarts in minimum error rate training for statistical machine translation. In Proceedings of the International Conference on Computational Linguistics (COLING) 2008, Manchester, August.

Franz Josef Och, Daniel Gildea, and Sanjeev Khudanpur et al. 2004. Final report of johns hopkins 2003 summer workshop on syntax for statistical machine translation (revised version). Technical report, February 25.

Franz Josef Och. 2003. Minimum error rate training for statistical machine translation. In Proceedings of the 41th Annual Meeting of the Association for Computational Linguistics (ACL), Sapporo, July.

William H. Press, Saul A. Teukolsky, William T. Vetterling, and Brian P. Flannery. 2002. Numerical Recipes in $\mathrm{C}++$. Cambridge University Press, Cambridge, UK.
Richard Zens and Hermann Ney. 2004. Improvements in phrase-based statistical machine translation. In Proceedings of Human Language Technology Conference / North American Chapter of the $A C L$, Boston, May.

Richard Zens, Sasa Hasan, and Hermann Ney. 2007. A systematic comparison of training criteria for statistical machine translation. In Proceedings of the 2007 Conference on Empirical Methods in Natural Language Processing (EMNLP), Prague, Czech Republic. 